\title{
INTEGRABILITY AND LINEARIZABILITY OF A FAMILY OF THREE-DIMENSIONAL QUADRATIC SYSTEMS
}

\author{
WALEED AZIZ ${ }^{1}$, AZAD AMEN ${ }^{1}$ AND ${ }^{*} \mathrm{CHARA} \mathrm{PANTAZI}^{2}$ \\ ${ }^{1}$ Department of Mathematics, Salahaddin University-Erbil, Kurdistan region-Iraq \\ ${ }^{2}$ Departament de Matemàtiques, Universitat Politècnica de Catalunya, Spain.
}

\begin{abstract}
We provide necessary and sufficient conditions for both integrability and linearizability of a three dimensional vector field with quadratic nonlinearities. For our investigation we consider the case of $(1:-2: 1)$-resonance at the origin and in general non of the axes planes is invariant. Hence, we deal with a nine parametric family of quadratic systems. Some techniques like Darboux method are used to prove the sufficiency of the obtained conditions. For a particular three parametric subfamily we provide conditions to guarantee the non existence of a polynomial first integral.
\end{abstract}

\section{Introduction AND Statement of the main RESUlts}

The problem of integrability is one of the most difficult problems in the qualitative theory of differential equations. For a three dimensional system, two independent first integrals are required in order the system to be completely integrable. In this case the trajectories of the system are completely determined by the two first integrals. To prove the existence or non existence of a first integral in general is a very hard problem, especially when the system depends on parameters. So, during the years many technics have been developed relating to first integrals, like Lie symmetries [32], Darboux theory of integrability [13, 14], Painlevé analysis [8], Differential Galois Theory $[28,35]$, among many others.

In this work we deal with the local integrability and linearizability problem at the origin of the three dimensional systems

$$
\begin{aligned}
& \dot{x}=P=\lambda x+a x y+b x z+c y z, \\
& \dot{y}=Q=\mu y+d x y+e x z+f y z, \\
& \dot{z}=R=\nu z+g x y+h x z+k y z,
\end{aligned}
$$

with $\lambda=1, \mu=-2$ and $\nu=1$ and the nine parameters $a, b, c, d, e, f, g, h$ and $k$ are real.

E-mail address: waleed.aziz@su.edu.krd, azad.amen@su.edu.krd, chara.pantazi@upc.edu.

2010 Mathematics Subject Classification. Primary 37K10 Secondary 34A05.

Key words and phrases. Integrability, linearizability, first integral, Jacobi multiplier, Darboux function. 
In the particular case where the axes planes $x=0, y=0$ and $z=0$ are invariants of system (1) then we obtain a three dimensional Lotka-Volterra system. Such systems have been invistigated by several authors like Bobienski and Żołądek [5], Aziz [1], Aziz and Christopher [3], Cairo and Llibre [10, 11], Buzzi et al. [9], Murza and Teruel [31], Basov and Romanovski [4], Christodoulides and Damianou [12], Gao and Liu, [19], Gonzalez and Peralta[20], Moulin-Ollagnier [30]) among others.

There is a big challenge to understand the mechanisms of integrability of non Lotka-Volterra systems. Some authors have been studied some non Lotka-Volterra systems: Dukarić et al. [16] gave necessary and sufficient conditions for integrability and linearizability for a family of three dimensional quadratic systems. They used mainly Darboux theory of integrability and other methods to prove the sufficiency of their conditions. Romanovski and collaborators in $[17,18]$ investigated the integrability problem with $(0:-1: 1)$ resonance at the origin. Local integrability and linearizability of a quadratic three dimensional family with $(1:-1: 1)$ resonance is considered in [2]. All these works aim to understand the underlying mechanism of the general problem of local integrability. Our goal is to contribute in this direction.

The main result of the paper is the following theorem.

Theorem 1.1. Consider the three dimensional system (1) with $(\lambda, \mu, \nu)=(1,-2,1)$. The origin is integrable if and only if one of the following conditions are satisfied:

1) $2 b^{2}-4 b f-c e+2 f^{2}=k=h=g=d=a=0$

2) $g=f=e=d=c=b=0$

3) $h=g=f=d=c=b=a+k=0$

4) $k=g=f=e=d+h=b=a=0$

5) $h=g=f=e=d=c=0$

6) $g=f=e=d=c=a-k=0$

7) $k=g=c=a=0$

8) $b d+2 b h+f h=a f-2 b k-f k=a d+2 a h-d k=g=e=c=0$

9) $2 d^{2}-4 d h-e g+2 h^{2}=k=f=c=b=a=0$

10) $h=f=e=d=b=0$

11) $k=h=e=d=c=b+f=a=0$

Moreover, the system is linearizable if and only if conditions above holds except condition (8).

In general, for parametric families of polynomial differential systems the problem of existence or non-existence of polynomial first integrals is a very difficult problem. In this direction there are some works for three dimensional Lotka-Volterra systems, see for example [22, 26, 29, 23]. Some other investigations on polynomial first integrals of general three dimensional systems can be found in $[10,25,27]$. 
We now consider the particular systems (1) where $c=e=g=f=d=0$ and $k=a$. Then system (1) becomes the Lotka-Volterra system

$$
\begin{aligned}
& \dot{x}=x(1+a y+b z), \\
& \dot{y}=-2 y, \\
& \dot{z}=z(1+h x+a y) .
\end{aligned}
$$

Note that system (2) corresponds to case 6) of Theorem 1.1. Our result about polynomial first integrals is the following theorem.

Theorem 1.2. If (i) $h=0$ and $a \neq 0$ or (ii) $h a \neq 0$ then the three parametric family (2) has no polynomial first integrals.

Remark 1. (i) System (1) under the condition 6) of Theorem 1.1 has no polynomial first integrals. The same holds for cases 2) and 5) when $k=a$.

(ii) If $a=h=0$, then $y z^{2}$ is a polynomial first integral of family (2). The same holds for cases 4 ) and 6) of Theorem 1.1.

The paper is organized as follows: In Section 2, some basic definitions and known results are given. Additionally, we explain some mechanisms to find necessary conditions for both integrability and linearizability. The proofs of Theorems 1.1 and 1.2 are given in Section 3. At the end, in Section 4 we present the conclusions of this work.

\section{KNOWN RESULTS}

Here we present the basic definitions and theorems that we use in order to understand the proofs and the results of the papers. Our purpose is to present a self contained paper.

2.1. Basic definitions. We say that the eigenvalue $\lambda$ is a resonant eigenvalue at the origin of system (1) if satisfies the following arithmetic condition

$$
\lambda n_{1}+\mu n_{2}+\nu n_{3}-\lambda=0, \quad|n|=n_{1}+n_{2}+n_{3} \geq 2
$$

for some non-negative integers $n_{i} \in \mathbb{N}_{0}=\{0,1,2, \ldots\}$. The natural number $|n|=n_{1}+n_{2}+n_{3}$ is the order of the resonance and the monomial $X^{n}=x^{n_{1}} y^{n_{2}} z^{n_{3}} e_{1}$ is a resonant monomial. The coefficient of the monomial $X^{n}$ in the system (1) is called a resonant coefficient and the corresponding term is called a resonant term. If condition (3) does not hold then the eigenvalue $\lambda$ is said to be nonresonant.

The Poincaré domain is the convex hull of the eigenvalues that does not contain the origin inside or on the boundary. The Siegel domain is the complement of the Poincaré domain.

We choose the eigenvalues $\lambda, \mu$ and $\nu$ to be in the Siegel domain and having two independent resonances. So, without loss of generality, (maybe considering a scaling of time) we can assume $\lambda, \mu, \nu \in \mathbb{Z}$ such that $\operatorname{gcd}(\lambda, \mu, \nu)=1$, and $\lambda, \nu>0$ and $\mu<0$. In this case we say that the origin has $(\lambda: \mu: \nu)$-resonance. 
Now for system (1) we consider its associated vector field

$$
\mathcal{X}=P \frac{\partial}{\partial x}+Q \frac{\partial}{\partial y}+R \frac{\partial}{\partial z} .
$$

We denote by $U$ an open neighborhood of the origin of $\mathbb{C}^{3}$. The non-constant analytic function $H: U \rightarrow \mathbb{C}$ is a local first integral of system (1) if it is constant on all solutions of the system contained in $U$. Hence, in all the points of $U$ we have

$$
\mathcal{X}(H)=P \frac{\partial H}{\partial x}+Q \frac{\partial H}{\partial y}+R \frac{\partial H}{\partial z}=0 .
$$

Consider that the two local first integrals $H_{1}$ and $H_{2}$ are defined in $U$. We say that are independent if their gradients are linear independent in $\mathbb{C}^{3}$ except perhaps in a set of measure zero.

A function $M: U \rightarrow \mathbb{C}$ is a Jacobi multiplier of $\mathcal{X}$ if it satisfies the equation

$$
\mathcal{X}(M)=-M \operatorname{div}(\mathcal{X}) \quad \text { in } \quad U
$$

where $\operatorname{div}(\mathcal{X})=\partial P / \partial x+\partial Q / \partial y+\partial R / \partial z$. If $M: U \rightarrow \mathbb{C}$ is a Jacobi multiplier then $1 / M: U \backslash\{M=$ $0\} \rightarrow \mathbb{C}$ is the inverse Jacobi multiplier.

Given a polynomial $F \in \mathbb{C}[x, y, z]$, a surface $F=0$ is called an invariant algebraic surface of system (1), if the polynomial $F$ satisfies the partial differential equation

$$
\dot{F}=\mathcal{X}(F)=P \frac{\partial F}{\partial x}+Q \frac{\partial F}{\partial y}+R \frac{\partial F}{\partial z}=C_{F} F
$$

for some polynomial $C_{F} \in \mathbb{C}[x, y, z]$. Such a polynomial $C_{F}$ is called the cofactor of the invariant algebraic surface $F=0$ and for system (1) has at most degree one.

To complete the study of the first integrals of parametric families, we will need to consider the exponential factors which appears when invariant algebraic surfaces collide. Let $E(x, y, z)=$ $\exp (f(x, y, z) / g(x, y, z))$ where $f, g \in \mathbb{C}[x, y, z]$ and $\operatorname{gcd}(f, g)=1$, then $E$ in an exponential factor if

$$
\mathcal{X}(E)=C_{E} E
$$

for some polynomial $C_{E}$ of degree at most one. The polynomial $C_{E}$ is called the cofactor of the exponential factor $E$.

A Darboux function is a function of the form,

$$
\prod F_{i}^{\lambda_{i}} \prod E_{j}^{\mu_{j}}
$$

where the $F_{i}$ are invariant algebraic surfaces of the system, and $E_{j}=\exp \left(f_{j} / g_{j}\right)$ are exponential factors. Given a Darboux function, we can compute

$$
\mathcal{X}\left(\prod F_{i}^{\lambda_{i}} \prod E_{j}^{\mu_{j}}\right)=\left(\sum \lambda_{i} C_{F_{i}}+\mu_{j} C_{E_{j}}\right) \prod F_{i}^{\lambda_{i}} \prod E_{j}^{\mu_{j}} .
$$

Clearly, the Darboux function is a non-trivial first integral of the vector field $\mathcal{X}$ if and only if the cofactors $C_{F_{i}}$ and $C_{E_{i}}$ are linearly dependent. This is a classical result due to Darboux [13]. 
Sometimes it is extremely hard to calculate explicitly the expressions of the first integrals of system (1). Alternatively, we say that system (1) is integrable at the origin if there exists a change of coordinates of the form

$$
X=x(1+O(x, y, z)), \quad Y=y(1+O(x, y, z)), \quad Z=z(1+O(x, y, z)),
$$

transforming system (1) to

$$
\dot{X}=\lambda X m, \quad \dot{Y}=\mu Y m, \quad \dot{Z}=\nu Z m,
$$

with $m=m(X, Y, Z)=1+O(X, Y, Z),[6,7]$. Note that system (1) is orbitally equivalent to the linear system (6). Some times we can choose $m \equiv 1$ and then we say the system (1) is linearizable.

It is clear that system (6) admits the analytic first integrals

$$
\phi=X^{-\mu} Y^{\lambda} \quad \text { and } \quad \psi=Y^{\nu} Z^{-\mu},
$$

which pull back to the first integrals of system(1)

$$
\phi_{1}=x^{-\mu} y^{\lambda}+O(x, y, z), \quad \text { and } \quad \phi_{2}=y^{\nu} z^{-\mu}+O(x, y, z) .
$$

Conversely, given two such first integrals, it is easy to construct a change of coordinates such that $\phi$ and $\psi$ expressed in these new coordinates satisfy (7), and hence the transformed system is of the form (6) for some $m$.

2.2. Known theorems. In some cases it is easy to deduce the linearizability of a singularity using the integrability.

Theorem 2.1. [3] Assume that system (1) is a Lotka-Volterra system. Consider that it is integrable and there exists a function $\xi=x^{\alpha} y^{\beta} z^{\gamma}(1+O(x, y, z))$ such that $X(\xi)=k \xi$ for some constant $k=\alpha \lambda+\beta \mu+\gamma \nu$, then the system is linearizable.

Additionally, we will use the following theorem.

Theorem 2.2. [2] Consider the system

$$
\dot{X}=\lambda X, \quad \dot{y}=y(-\mu+A(X, Z))+B(X, Z), \quad \dot{Z}=\nu Z,
$$

where $\lambda, \mu, \nu \in \mathbb{Z}^{+}$. The system is linearizable if there exist functions $\alpha$ and $\gamma$ such that $\dot{\alpha}+\gamma B=$ $-\mu \alpha$ and $\dot{\gamma}+\gamma A=0$, where $A(X, Z)$ and $B(X, Z)$ are functions of $X$ and $Z$.

The following result establish the importance of the Jacobi multipliers, see [21, 24].

Theorem 2.3. [21, 24] Consider the differential system

$$
\frac{d \mathbf{x}}{d t}=\mathbf{P}(\mathbf{x}), \quad \mathbf{x}=\left(\mathbf{x}_{1}, \ldots, \mathbf{x}_{\mathbf{n}}\right) \in \mathbb{R}^{\mathbf{n}},
$$

with $\mathbf{P}(x)=\left(P_{1}(x), \ldots, P_{n}(x)\right)$ and $P_{i} \in \mathbb{R}\left[x_{1}, \ldots, x_{n}\right]$ for $i=1, \ldots, n$. Assume that it admits a Jacobi multiplier and $n-2$ first integrals functionally independent. Then the system admits an additional first integral functionally independent with the previous $n-2$ first integrals. 
2.3. Mechanisms for integrability and linearizability. In this subsection, we present a complete classification of integrability and linearizability conditions of the three dimensional system (1) with $(1:-2: 1)$ resonant critical point at the origin. Hence, we consider the system

$$
\begin{aligned}
& \dot{x}=P=x+a x y+b x z+c y z, \\
& \dot{y}=Q=-2 y+d x y+e x z+f y z, \\
& \dot{z}=R=z+g x y+h x z+k y z .
\end{aligned}
$$

Our main objective along this section is to find conditions on the parameters such that system (1) possesses the two independent first integrals

$$
\phi_{1}=x^{2} y+O(x, y, z) \quad \text { and } \quad \phi_{2}=y z^{2}+O(x, y, z)
$$

In general, even thought the necessary conditions of integrability are obtained, their sufficiency is very difficult to be proved. We have used mainly the Darboux theory of integrability and also some properties of linearizable nodes of two dimensional systems. In the particular cases where system (1) becomes Lotka-Volterra we apply specific results like Theorem 2.1.

Linearizable node. Sometimes, we can choose a coordinate system so that two of the variables decouple to give a linearizable node at the origin or can be brought to normal form via an analytic change of coordinates. In particular, a node with two analytic separatrices can have no resonant terms in its normal form and so must be analytically linearizable. If this is so, it just remains to find a linearizing transformation for the third variable via some simple power series arguments. Since this new system is linearizable, we can find two first integrals which we can pull back to first integrals of the original system.

Integrability and linearizability conditions of system (3). We will first seek necessary conditions for integrability and linearizability at the origin of system (9). So we consider $\phi_{1}$ and $\phi_{2}$ in the form (10) and we write them as power series up to degree 15. In order to find the necessary conditions we compute the obstructions to form first integrals which are known as resonant focus quantity, for more details see [16]. Then, a factorized Gröbner basis was found using the Computer Algebra system Reduce. Finally, the minAssGTZ algorithm of the Computer Algebra system Singular $[15,33]$ was used to check that the conditions found were irreducible. To prove the sufficiency of the conditions, we exhibit first integrals of the form (10).

For linearizability, we proceeded similarly: In order to find necessary conditions we compute the conditions for the existence of a linearizing change of coordinates up to order 13 . Then by exhibiting a linearizing change of coordinates we prove the sufficiency. In this case, the first integrals of system (9) can be obtained easily by pulling back the first integrals of the linearized system (10). For more detail, one can consult $[2,3]$. 


\section{Proofs of Theorems 1.1 And 1.2}

Proof of Theorem 1.1. Case 1: System (9) becomes

$$
\dot{x}=b x z+c y z+x, \quad \dot{y}=-2 y+e x z+f y z, \quad \dot{z}=z,
$$

and $e c=2(b-f)^{2}$.

Subcase-1 $b \neq f$. In this subcase system (11) has the invariant algebraic surfaces $\ell_{1}=z=0$, $\ell_{2}=2 x+2(b-f) x z+c y z=0$ and $\ell_{3}=2 x+c y z+4(f-b) x z+3 c(f-b) y z^{2}+\left(3 b^{2}-6 b f+3 f^{2}\right) x z^{2}=0$ and an exponential factor $\ell_{4}=\exp (z)$ with respective cofactors $1,1+(2 b-f) z, 1+(2 f-b) z$ and $z$. System (11) has the two independent Darboux first integrals

$$
H_{1}(x, y, z)=\ell_{1}^{-1} \ell_{2} \ell_{4}^{f-2 b}, \quad H_{2}(x, y, z)=\ell_{1}^{-1} \ell_{3} \ell_{4}^{b-2 f} .
$$

Additionally, the change of coordinates $(X, Y, Z)=\left(\ell_{2} \ell_{4}^{f-2 b}, \ell_{3} \ell_{4}^{b-2 f}, \ell_{1}\right)$ yields to the linear system

$$
\dot{X}=X, \quad \dot{Y}=Y, \quad \dot{Z}=Z,
$$

and the origin of system (12) is in the Poincaré domain. Then, we can pull back the first integrals $\tilde{\Phi}=X Y^{-1}$ and $\tilde{\Psi}=Y Z^{-1}$ of system (12) to the first integrals of system (11) of the desired form $\phi_{1}=x^{2} y(1+O(x, y, z))$ and $\phi_{2}=y z^{2}(1+O(x, y, z))$.

Subcase-2 $b=f$. Then condition $2 b^{2}-4 b f-c e+2 f^{2}=0$ yields to $c e=0$.

First consider $c \neq 0$ and $e=0$. Then system (11) has the two independent Darboux first integrals

$$
H_{1}(x, y, z)=y z^{2} \exp (-f z), \quad H_{2}(x, y, z)=\frac{2 x+c y z}{z \exp (f z)} .
$$

The change of coordinates $(X, Y, Z)=((c y z+2 x) \exp (-f z), \quad y \exp (-f z), \quad z)$ linearizes the system.

Now consider $c=0$ and $e \neq 0$. Then system (11) has the Jacobi multipliers $M_{1}=1 /(\exp (z))^{2 f}$ and $M_{2}=z^{2} / x^{2}$. Additionally, has the first integral $H_{1}(x, y, z)=\sqrt{M_{2} / M_{1}}=z \exp (f z) / x$.

The change of coordinates $(X, Y, Z)=(x \exp (-f z), \quad(-4 y+e x z) \exp (-f z), \quad z)$ linearizes the system.

At the end consider $c=e=0$. Then system (11) is a Lotka-Volterra system and has the two independent Darboux first integrals

$$
H_{1}(x, y, z)=\frac{z \exp (f z)}{x}, \quad H_{2}(x, y, z)=\frac{y z^{3}}{x} .
$$

The change of coordinates $(X, Y, Z)=(2 x \exp (-f z), \quad y \exp (-f z), \quad z)$ linearizes the system.

Case 2: In this case system (9) reduces to the Lotka-Volterra system

$$
\dot{x}=x(1+a y), \quad \dot{y}=-2 y, \quad \dot{z}=z(1+h x+k y) .
$$

System (13) has the invariant surfaces $x=0$ and $y=0$ with cofactors $K_{1}=a y+1$ and $K_{2}=-2$, respectively. Additionally, system (13) admits the $\operatorname{exponential~factor} \exp (y)$ with cofactor $K_{3}=$ $-2 y$. 
For $a \neq 0$ system (13) has the Darboux first integral $H(x, y, z)=x^{2} y(\exp (y))^{a}$ and the Darboux Jacobi multiplier $M_{1}=(x y z)^{-1}$. According to Theorem 2.3, system (13) admits an additional first integral.

The transformation $(X, Y)=\left(x \exp \left(\frac{a}{2} y\right), y\right)$ linearizes the first two equations of system (13) and can be written as

$$
\dot{X}=X, \quad \dot{Y}=-2 Y .
$$

Now, a change of coordinates $Z=z \exp (-\phi)$ with $\phi$ an analytic function of the variables $X$ and $Y$ will give $\dot{Z}=Z$ if and only if

$$
\dot{\phi}(X, Y)=X \frac{\partial \phi}{\partial X}-2 Y \frac{\partial \phi}{\partial Y}=h x(X, Y)+k y(Y) .
$$

We have

$$
x=X \exp \left(-\frac{a}{2} Y\right)=\sum_{n \geq 1} \frac{1}{(n-1) !}\left(-\frac{a}{2}\right)^{n-1} X Y^{n-1},
$$

and note that $x$ contains no terms of the form $\left(X^{2} Y\right)^{n}$. This guarantees that the solution of relation (14) is analytic.

For $a=0$ system (11) has the two independent Darboux first integrals

$$
H_{1}(x, y, z)=x^{2} y, \quad H_{2}(x, y, z)=z^{2} y \exp (-2 h x+k y) .
$$

The change of coordinates $(X, Y, Z)=\left(x, y, z \exp \left(-h x+\frac{k}{2} y\right)\right)$ linearizes the last equation.

Case 3: System (9) becomes

$$
\dot{x}=x(1-k y), \quad \dot{y}=-2 y+e x z, \quad \dot{z}=z(1+k y) .
$$

System (15) is a divergence free system and has the Darboux first integral

$$
H_{1}(x, y, z)=\frac{x^{2} \exp (e x z-2 y)}{z^{2}} .
$$

The change of variables $X=x \exp \left(\frac{k}{4}(e x z-2 y)\right), \quad Z=z \exp \left(-\frac{k}{4}(e x z-2 y)\right)$ will gives $\dot{X}=X$, $\dot{Z}=Z$. Note that the linear equation $\dot{y}=-2 y+e X Z$ has a particular solution

$$
y_{1}=\frac{1}{4} e X Z \text {. }
$$

The substitution $y=Y+y_{1}$ gives $\dot{Y}=-2 Y$. Hence, system (15) is linearizable and so has the two independent first integrals $\phi=X^{2} Y$ and $\psi=Y Z^{2}$.

Case 4: In this case system (9) can be written

$$
\dot{x}=x+c y z, \quad \dot{y}=y(-2-h x), \quad \dot{z}=z(1+h x) .
$$


System (16) is a divergence free system.

For $h \neq 0$ has the two independent first integrals

$$
H_{1}(x, y, z)=\frac{y z^{2}}{x+\exp (x+c y z)^{h}}, \quad H_{2}(x, y, z)=\frac{z}{(2 x+c y z)(\exp (x+c y z))^{h}} .
$$

The change of coordinates

$$
(X, Y, Z)=(x+c y z / 2, y \exp (h(x+c y z)), z \exp (-h(x+c y z))) .
$$

linearizes system (16).

For $h=0$ system (16) has the two independent first integrals

$$
H_{1}(x, y, z)=y z^{2}, \quad H_{2}(x, y, z)=\exp (\sqrt{y}(2 x+c y z)) .
$$

The change of coordinates $(X, Y, Z)=(x+c y z / 2, y, z)$ linearizes the system.

Case 5: System (9) takes the form

$$
\dot{x}=x(1+a y+b z), \quad \dot{y}=-2 y, \quad \dot{z}=z(1+k y) .
$$

This case is dual with Case 2 under the transformations $x \mapsto z, a \mapsto k$, and $b \mapsto h$.

Case 6: The new subsystem is a Lotka-Volterra system and takes the form

$$
\dot{x}=x(1+k y+b z), \quad \dot{y}=-2 y, \quad \dot{z}=z(1+h x+k y),
$$

System (17) has the first integral $H_{1}(x, y, z)=y(h x-b z)^{2} \exp (k y)$ and the Jacobi multiplier $M=(x y z)^{-1}$. Now Thoerem 2.3, guarantee the existence of another first integral independent of $H_{1}$.

Since $\xi=y$ satisfy $\dot{\xi}=-2 \xi$, then by Theorem 2.1 , system (17) is linearizable.

Case 7: System (9) becomes

$$
\dot{x}=x(1+b z), \quad \dot{y}=-2 y+d x y+e x z+f y z, \quad \dot{z}=z(1+h x) .
$$

System (18) has the Darboux first integral

$$
H_{1}(x, y, z)=\frac{z \exp (b z-h x)}{x} .
$$

Note that the first and third equation correspond to a linearizable node. Hence, there exists a change of coordinates of the form $X=x(1+O(x, z))$ and $Z=z(1+O(x, z))$ such that the system becomes

$$
\dot{X}=X, \quad \dot{y}=y(-2+d x(X, Z)+f x(X, Z))+e x(X, Z) z(X, Z), \quad \dot{Z}=Z .
$$

Consider $A(X, Z)=d x(X, Z)+f x(X, Z)$ and $B(X, Z)=e x(X, Z) z(X, Z)$. In order to apply Theorem 2.2 we should prove that the system $\dot{\alpha}+\gamma B=-2 \alpha$ and $\dot{\gamma}+\gamma A=0$, has solutions. Consider $\gamma=\exp (-\zeta)$ with $\zeta=\sum_{i+j>0} \zeta_{i j} X^{i} Z^{j}$. Then from relation $\dot{\gamma}+\gamma A=0$ we have that 
$\zeta_{i j}=a_{i j} /(i+j)$ with $i+j>0$ and the convergence of $A$ guarantees the convergence of $\zeta$. Now consider $\alpha=\sum_{i+j>0} \alpha_{i j} X^{i} Y^{j}$ and $\gamma B=\sum_{i+j>0} \delta_{i j} X^{i} Z^{j}$. From relation $\dot{\alpha}+\gamma B=-2 \alpha$ we have that $\alpha_{i j}=-\delta_{i j} /(i+j+2)$. So, the convergence of $\gamma B$ guarantees the convergence of $\alpha$. Hence, according to Theorem 2.2 system (19) is linearizable.

Case 8: System (9) is a Lotka-Volterra system and can be written as

$$
\dot{x}=x(1+a y+b z), \quad \dot{y}=y(-2+d x+f z), \quad \dot{z}=z(1+h x+k y) .
$$

If $b \neq 0$ and $f \neq 0$, then due to Theorem 1.1(8) we have that $a=k(2 b+f) / f$ and $d=-(2 b+f) h / b$ and the exponential factor $E=\exp \left(h x+\frac{b k}{f} y-b z\right)$ has cofactor $L_{E}=h x-\frac{2 b k}{f} y-b z$. So we can construct the two independent Darboux first integrals

$$
\phi_{1}(x, y, z)=x^{2} y E^{2+\frac{f}{b}} \quad \text { and } \quad \phi_{2}(x, y, z)=y z^{2} E^{\frac{f}{b}} .
$$

When $b \neq 0$ and $f=0$, then due to Theorem 1.1(8) it must be $k=0$ and $d=-2 h$. The exponential factor $E=\exp \left(h x+\frac{1}{2} a y-b z\right)$ has cofactor $L_{E}=h x-a y-b z$ and we obtain the Darboux first integrals

$$
\phi_{1}(x, y, z)=x^{2} y E^{2} \quad \text { and } \quad \phi_{2}(x, y, z)=y z^{2} .
$$

If $b=0$ and $f \neq 0$, then it must be $h=0$ and $k=a$ and the exponential factor $E=$ $\exp (a y-d x-f z)$ has cofactor $L_{E}=-d x-2 a y-f z$. The two independent first integrals are

$$
\phi_{1}=x^{2} y E \quad \text { and } \quad \phi_{2}=y z^{2} E .
$$

If $b=f=0$, then we can assume $d \neq 0$ since otherwise we are in Case 2 of Theorem 1.1. Then it must be $k=a(d+2 h) / d$ and the exponential factor $E=\exp (a y-d x)$ has cofactor $L_{E}=-d x-2 a y$. Then we obtain the two independent first integrals

$$
\phi_{1}=x^{2} y E \quad \text { and } \quad \phi_{2}=y z^{2} E^{\frac{k}{a}} .
$$

Case 9: System (9) becomes

$$
\dot{x}=x, \quad \dot{y}=-2 y+d x y+e x z, \quad \dot{z}=z+g x y+h x z .
$$

Consider first that $g=0$. Then by relation $2 d^{2}-4 d h-e g+2 h^{2}=0$ we have $d=h$. In this case system (21) becomes

$$
\dot{x}=x, \quad \dot{y}=-2 y+e x z+h x y, \quad \dot{z}=z(1+h x),
$$

and has the two independent first integrals

$$
H_{1}(x, y, z)=\frac{z \exp (-h x)}{x}, \quad H_{2}(x, y, z)=x^{2}(-e x z+4 y) \exp (-h x) .
$$

Additionally, the algebraic surface $\ell=4 y-e x z=0$ is invariant with cofactor $K=-2+h x$. The change of coordinates $(X, Y, Z)=(x, \ell \exp (-h x), z \exp (-h x))$ linearizes the system. 
Now consider that $g \neq 0$. Then using relation $2 d^{2}-4 d h-e g+2 h^{2}=0$, system $(21)$ becomes

$$
\dot{x}=x, \quad \dot{y}=-2 y+d x y+\frac{2(d-h)^{2}}{g} x z, \quad \dot{z}=z+g x y+h x z,
$$

and for $d \neq h$ has the two surfaces $f_{1}=2(d-h) x z-g x y-2 z=0, \quad f_{2}=2 z+g x y+4(d-h) x z+$ $3 g(d-h) x^{2} y+3(d-h)^{2} x^{2} z=0$ and $f_{3}=x=0$ invariants with cofactors $K_{1}=1+(2 h-d) x$, $K_{2}=1+(2 d-h) x$ and $K_{3}=1$ respectively. Additionally, system (22) admits the exponential factor $E=\exp (x)$ with cofactor $K=x$.

Moreover, system (22) is integrable with the two independent Darboux first integrals

$$
H_{1}(x, y, z)=f_{1}^{-1} f_{3} E^{2 h-d}, \quad H_{2}(x, y, z)=f_{1}^{-1} f_{2} E^{3(h-d)} .
$$

Additionally, system (22) admits the Jacobi multiplier $M=E^{-h-d}$. A linearizing change of coordinates is given by

$$
(X, Y, Z)=\left(x, f_{1} E^{d-2 h}, f_{2} E^{(h-2 d)}\right) .
$$

For $d=h$ and since $g \neq 0$ we have that $e=0$. System (22) admits the two independent first integrals

$$
H_{1}(x, y, z)=y x^{2} \exp (-h x), \quad H_{2}(x, y, z)=\frac{\left(y x^{2} g+2 z x\right) \exp (-h x)}{x^{2}} .
$$

The change of coordinates

$$
(X, Y, Z)=\left(x, \quad y^{2}\left(\mathrm{e}^{-3 h x}\right) x(g x y+2 z), \quad y^{2} x^{4}\left(\mathrm{e}^{-3 h x}\right)(g x y+2 z)\right),
$$

linearizes the system.

Case 10: In this case system (9) becomes

$$
\dot{x}=x+a x y+c y z, \quad \dot{y}=-2 y, \quad \dot{z}=z+g x y+k y z,
$$

and has the surfaces $f_{1}=y=0$ and $f_{2}=c z^{2}+(a-k) x z-g x^{2}=0$ invariants with cofactors $K_{1}=-2$ and $K_{2}=a y+k y+2$, respectively. Additionally, system (23) admits the exponential factor $E=\exp (y)$ with cofactor $K=-2 y$.

For $a \neq-k$ system (23) has the first integral $H_{1}=f_{2}^{2} f_{1}^{2} E^{a+k}$ and the Jacobi multiplier $M=$ $E^{(a+k) / 2}$. Hence, by Theorem 2.3 admits an additional first integral.

For $a=-k$ system (23) is a divergence free system and has the first integral $H=f_{1} f_{2}$.

For both cases the system will linearize under the transformation

$$
(X, Y, Z)=\left(\ell_{1} E^{\frac{\alpha_{1}}{2}}, y, \ell_{2} E^{\frac{\alpha_{2}}{2}}\right)
$$


where $\ell_{1}=r_{1} x+c z$ and $\ell_{2}=r_{2} x+c z$ such that

$$
\begin{aligned}
r_{1} & =\frac{1}{2}\left(a-k+\sqrt{a^{2}-2 a k+4 c g+k^{2}}\right), \\
r_{2} & =\frac{1}{2}\left(a-k-\sqrt{a^{2}-2 a k+4 c g+k^{2}}\right), \\
\alpha_{1} & =\frac{1}{2}\left(a+k+\sqrt{a^{2}-2 a k+4 c g+k^{2}}\right), \\
\alpha_{2} & =\frac{1}{2}\left(a+k-\sqrt{a^{2}-2 a k+4 c g+k^{2}}\right) .
\end{aligned}
$$

Case 11: System (9) becomes

$$
\dot{x}=x(b z+1), \quad \dot{y}=-y(b z+2), \quad \dot{z}=g x y+z,
$$

and is a divergence free system. System (24) has the surfaces $f_{1}=x=0, f_{2}=y=0$ and $f_{3}=z+\frac{g}{2} x y=0$ invariants with cofactors $K_{1}=b z+1, K_{2}=-b z-2$ and $K_{3}=1$ respectively. Additionally, admits the exponential factor $E=\exp (z+g x y)$ with cofactor $K=z$. System (24) has the Darboux first integrals $H_{1}=f_{1} f_{2} f_{3}$ and $H_{2}=E^{b} f_{3} / f_{1}$.

The transformation

$$
(X, Y, Z)=\left(f_{1} E^{-b}, f_{2} E^{b}, f_{3}\right)
$$

linearizes the system.

Proof of Theorem 1.2. We assume that $H=H(x, y, z)$ is a polynomial first integral of system (2). Then it must satisfy

$$
x(1+a y+b z) \frac{\partial H}{\partial x}-2 y \frac{\partial H}{\partial y}+z(1+h x+a y) \frac{\partial H}{\partial z}=0 .
$$

We can write $H=\sum_{i=0}^{n} H_{i}(y, z) x^{i}$, where for each $i, H_{i}(y, z)$ is a polynomial in the variables $y$ and $z$. Note that $H$ is of degree $n \in \mathbb{N} \cup\{0\}$ in the variable $x$. From (25) the terms in $x^{n+1}$ satisfy

$$
h z \frac{\partial H_{n}(y, z)}{\partial z}=0
$$

We distinguish the following two cases.

(i) $h=0$ and $a \neq 0$. First consider the case where the polynomial first integral does not depend on the variable $x$, so $n=0$ and $H=H_{0}(y, z)$. From relation (25) we have

$$
-2 y \frac{\partial H_{0}(y, z)}{\partial y}+z(1+a y) \frac{\partial H_{0}(y, z)}{\partial z}=0
$$

and the solution is

$$
H_{0}(y, z)=C_{0}\left(z \sqrt{y} \exp \left(\frac{a y}{2}\right)\right)
$$

with $C_{0}$ an arbitrary function in the variable $z \sqrt{y} \exp \left(\frac{a y}{2}\right)$. Since the first integral is a polynomial and $a \neq 0$ we have that $C_{0}$ must be zero and this means that $H=H_{0}(y, z)=0$, a contradiction. 
Now we consider that the first integral is of degree $n>0$ in the variable $x$ and so $H_{n} \neq 0$. The terms of $x^{n}$ in (25) satisfy

$$
n(1+a y+b z) H_{n}(y, z)-2 y \frac{\partial H_{n}(y, z)}{\partial y}+z(1+a y) \frac{\partial H_{n}(y, z)}{\partial z}=0 .
$$

The solution of the last linear partial differential equation is

$$
H_{n}(y, z)=C_{n}\left(z \sqrt{y} \exp \left(\frac{a y}{2}\right)\right) y^{\frac{n}{2}} \exp \left[\frac{n}{2}\left(-\exp \left(\frac{a y}{2}\right) \operatorname{erf}\left(\frac{\sqrt{2 a y}}{2}\right) \sqrt{2 a \pi y}-2\right) b z+\frac{n a y}{2}\right]
$$

and $C_{n}$ is an arbitrary function in the variable $z \sqrt{y} \exp \left(\frac{a y}{2}\right)$. Note that erf denotes the Error function which is not an elementary function, see for example [34]. Since $H_{n}$ is a polynomial and $a \neq 0$, then from the last expression of $H_{n}$ it must be $C_{n}=0$ and therefore $H_{n}=0$. This is a contradiction.

Note that for $b=0$ the solution of the partial linear differential equation is

$$
H_{n}(y, z)=C_{n}\left(z \sqrt{y} \exp \left(\frac{a y}{2}\right)\right) y^{\frac{n}{2}} \exp \left(\frac{n a y}{2}\right)
$$

and for $a \neq 0$ can never be a polynomial.

(ii) $h a \neq 0$. Then from (26) we have $H_{n}(y, z)=H_{n}(y)$. First consider that the polynomial first integral $H$ does not depend on the variable $x$, so consider that $n=0$. We have that $H=H_{0}(y, z)=$ $H_{0}(y)$. Then from equation $(25)$ we obtain that $\frac{d H_{0}(y)}{d y}=0$ and so the first integral $H=H_{0}$ must be a constant. This is a contradiction.

Now we consider the case where the first integral $H$ is of degree $n>0$ in the variable $x$ and so $H_{n} \neq 0$. Note that the terms of $x^{n}$ for $n>0$ in relation (25) satisfy

$$
n(1+a y+b z) H_{n}(y)-2 y \frac{d H_{n}(y)}{d y}+h z \frac{\partial H_{n-1}(y, z)}{\partial z}=0
$$

It is easy to see that equation (27) has the solution

$$
H_{n-1}(y, z)=\frac{\left[-n(1+a y) H_{n}(y)+2 y \frac{d H_{n}}{d y}\right] \ln (z)}{h}-\frac{n b}{h} z H_{n}(y)+C_{n}(y),
$$

where $C_{n}$ is an arbitrary function of $y$. Since $H_{n-1}(y, z)$ is a polynomial and additionally the function $C_{n}$ does not depend on the variable $z$, it must be

$$
-n(1+a y) H_{n}(y)+2 y \frac{d}{d y} H_{n}(y)=0,
$$

and so $H_{n}(y)=C_{1} \exp \left(\frac{a n y}{2}\right) y^{\frac{n}{2}}$, where $C_{1}$ is an arbitrary constant. But $H_{n}$ is a polynomial and since $n>0$ and $a \neq 0$ it must be $C_{1}=0$. This mean that $H_{n}=0$ and this is a contradiction because the first integral $H$ is of degree $n$ in the variable $x$.

Hence system (2) for cases (i) and (ii) has no polynomial first integrals. 


\section{Conclusion}

The problems of local integrability and linearizability are considered for the quadratic polynomial system (1) which depends on nine parameters. Complete quadratic system are not considered as the computation of resonant focus quantities is extremely complicated. We found necessary conditions for both integrability and linearizability at the origin via factorized Gröbner basis using Reduce and minAssGTZ algorithm in Singular [15, 33]. We use some special transformations and some techniques like Darboux method to prove the sufficiency of the obtained conditions. These results appears in the main Theorem 1.1. We also proved that for some of the cases of Theorem 1.1 system (1) has no polynomial first integrals, see Theorem 1.2.

\section{ACKNOWLEDGMENTS}

W. Aziz is financially supported by Ministry of Higher Education and Scientific Research-Iraq. C. Pantazi is partially supported by the Ministerio de Ciencia, Innovación y Universidades, Agencia Estatal de Investigación grant MTM2016-77278-P (FEDER), the Spanish MINECO-FEDER Grant PGC2018-098676-B-I00/AEI/FEDER/UE and by the Catalan Grant 2017SGR1049.

\section{REFERENCES}

[1] Aziz W., Analytic and Algebraic Aspects of Integrability for First Order Partial Differential Equations, Ph.D. Thesis, Plymouth University, 2013.

[2] Aziz W., Integrability and linearizability of three dimensional vector fields, Qual. Theory Dyn. Syst. 2014; 13: 197-213.

[3] Aziz W. and Christopher C., Local integrability and linearizability of three-dimensional Lotka-Volterra systems, Appl. Math. Comput. 2012; 219(8): 4067-4081.

[4] Basov V. V. and Romanovski V. G., First integrals of a three-dimensional system in the case of one zero eigenvalue, J. Phys. A: Math. Theor. 2010; 43: 1-8.

[5] Bobienski M. and Żołądek H., The three-dimensional generalized Lotka-Volterra systems, Ergodic Theory Dynam. Syst. 2005; 25: 759-791.

[6] Bruno A. D., Analytical form of differential equations, Transactions of the Moscow Mathematical Society, 1971; 25: $131-288$.

[7] Bruno A. D., Local Methods in Nonlinear Differential Equations, Springer Verlag, New York, 1989.

[8] Bountis T. C., Ramani A., Grammaticos B. and Dorizzi B., On the complete and partial integrability of non-Hamiltonian systems, Phys. A, 1984; 128: 268-288.

[9] Buzzi C. A., Santos R. A. T. and Llibre J., Final evolutions of a class of May-Leonard Lotka-Volterra systems, J. of Nonlinear Mathematical Physics, 2020; 27: 267-278.

[10] Cairó L., Darboux first integral conditions and integrability of the 3D Lotka-Volterra system, J. Nonlinear Math. Phys. 2000; 7(4): 511-531.

[11] Cairó L. and Llibre J., Darboux integrability for 3D Lotka-Volterra systems, J. Phys. A: Math. Gen. 2000; 33(12): 2395-2406.

[12] Christodoulides Y. T. and Damianou P. A., Darboux polynomials for Lotka-Volterra systems in three dimensions, J. Nonlinear Math. Phys. 2009; 16: 339-354. 
[13] Darboux G., De l'emploi des solutions particulières algébriques dans l'intégration des systèmes d'équations différentielles algébriques, C. R. Math. Acad. Sci. Paris 1878; 86: 1012-1014.

[14] Darboux G., Mémoire sur les équations différentielles algébriques du premier ordre et du premier degré (Mélanges), Bull. Sci. math. 2ème série, 1878; 2: 60-96; 123-144; 151-200.

[15] Decker W., Greuel G. M., Pfister G., Schönemann, H.: Singular 4-1-2 A computer algebra system for polynomial computations. http://www.singular.uni-kl.de 2019.

[16] Dukarić M., Oliveira R. and Romanovski V. G., Local Integrability and Linearizability of a (1: $-1:-1)$ Resonant Quadratic System, J Dyn Diff Equat. 2015; 27: 1-17.

[17] Hu Z., Aldazharova M., Aldibekov T. M. and Romanovski V. G., Integrability of 3-dim polynomial systems with three invariant planes, Nonlinear Dyn. 2013; 74: 1077-1092.

[18] Hu Z., Han M., Romanovski V. G., Local integrability of a family of three-dimensional quadratic systems, Physica D2013; 265: 78-86.

[19] Gao P., Liu Z., An indirect method of finding integrals for three-dimensional quadratic homogeneous systems, Phy. Lett. A 1998; 244: 49-52.

[20] Gonzalez-Gascon F. and Peralta Salas D., On first integrals of Lotka-Volterra systems, Phys. Lett. A 2000; 266: 336-340.

[21] Goriely A., Integrability and non integrability of dynamical systems, Advances Series in Nonlinear Dynamics 19 World Scientific PublishingCo., Inc., River Edge, NJ, 2001.

[22] Labrunie S., On the polynomial first integrals of the (a,b,c) Lotka-Volterra systems, J. Math. Phys. 1966; 37: $5539-5550$.

[23] Leach P. G. and Miritzis J., Analytic behaviour of competition among three species, J. Math. Anal. Appl. 2013; 399: 411-417.

[24] Llibre J., Yu J. and Zhang X., On the integrability and polynomial integrability of the Euler equations, Internat. J. Geom. Phys. 2015; 96: 36-41.

[25] Llibre J. and Valls C., On the polynomial first integral of the Hoyer systems, J. Nonlinear. Math. Phys. 2006; 13: $535-548$.

[26] Llibre J. and Valls C., Polynomial, rational and analytic first integrals for a family of 3-dimensional LotkaVolterra systems, Z. Angew. Math. Phys. 2011; 7: 76-777.

[27] Llibre J. and Valls C., Polynomial first integrals of the Chen and Lu systems, Internat. J. Bifur. Chaos Appl. Sci. Engrg. 2012; 22: 125-262.

[28] Morales-Ruiz J.J., Differential Galois theory and Non-integrability of Hamiltonian Systems, Progress in Mathematics 179, Birkhäuser, Basel 1999.

[29] Moulin-Ollagnier J., Polynomial first integrals of the Lotka-Volterra systems, Bull. Sci. Math. 1997; 121: 463476.

[30] Moulin-Ollagnier J., Liouvillian integration of the Lotka-Volterra systems, Qual. Theory Dynam. Syst. 2001; 2: 307-358.

[31] Murza A. C. and Teruel A. E., Global dynamics of a family of 3-D Lotka-Volterra systems, Dynamical Systems: An International Journal, 2010; 25: 269-284.

[32] Olver P. J., Applications of Lie groups to differential equations, Springer, New York, 1986.

[33] Pfister G., Decker W. and Schönemann H., A Singular 4-0-2 library for primary decomposition and radicals of ideals, 2015.

[34] Ritt J. F., Integration in Finite Terms, Columbia Univ. Press, New York 1948.

[35] Singer M. F., Liouvillian first integrals of differential equations, Trans. Amer. Math. Soc., 1992; 333: 673-688. 